Research Article

\title{
The Imbalance of B-Lymphocyte Subsets in Subjects with Different Glucose Tolerance: Relationship with Metabolic Parameter and Disease Status
}

\author{
Chao Deng, ${ }^{1,2}$ Yufei Xiang, ${ }^{1,2}$ Tingting Tan, ${ }^{1,2}$ Zhihui Ren, ${ }^{1,2}$ Chuqing Cao,, ${ }^{1,2}$ Bingwen Liu, ${ }^{1,2}$ \\ Gan Huang, ${ }^{1,2}$ Xiangbing Wang, ${ }^{3}$ and Zhiguang Zhou ${ }^{1,2}$ \\ ${ }^{1}$ Department of Metabolism \& Endocrinology, The Second Xiangya Hospital, Central South University, Changsha, \\ Hunan 410011, China \\ ${ }^{2}$ Key Laboratory of Diabetes Immunology, Central South University, Ministry of Education, National Clinical Research Center for \\ Metabolic Diseases, Changsha, Hunan 410011, China \\ ${ }^{3}$ Division of Endocrinology, Department of Medicine, Rutgers Robert Wood Johnson Medical School, New Brunswick, NJ, USA
}

Correspondence should be addressed to Zhiguang Zhou; zhouzg@hotmail.com

Received 3 January 2017; Revised 4 March 2017; Accepted 14 March 2017; Published 16 April 2017

Academic Editor: Craig S. Nunemaker

Copyright (c) 2017 Chao Deng et al. This is an open access article distributed under the Creative Commons Attribution License, which permits unrestricted use, distribution, and reproduction in any medium, provided the original work is properly cited.

B lymphocytes are involved in inflammation and are related to insulin resistance in obesity and type 2 diabetes (T2D). This study investigated the phenotype and frequency of B-lymphocyte subsets in subjects recently diagnosed with T2D $(n=60)$, impaired glucose regulation (IGR, $n=73$ ), and normal glucose tolerance (NGT, $n=169$ ) by flow cytometry. T2D subjects had an increased percentage of $\mathrm{CD} 19^{+} \mathrm{CD} 23^{+}$(B-2) cells and a decreased percentage of $\mathrm{CD} 19^{+} \mathrm{CD} 23^{-}$(B-1) cells attributing to $\mathrm{CD} 19^{+} \mathrm{CD} 23^{-} \mathrm{CD}^{-}$(B-1b) cells, but not $\mathrm{CD} 19^{+} \mathrm{CD} 23^{-} \mathrm{CD}^{+}$(B-1a) cells, compared to NGT and IGR subjects. The proportion of $\mathrm{CD} 19^{+} \mathrm{CD}^{+} \mathrm{CD} 1 \mathrm{~d}^{\text {hi }}$ (B10) cells did not differ between the IGR or T2D group and NGT controls. Of note, HbA1c and triglyceride showed a positive correlation with B-2 cells but an inverse correlation with B-1 and B-1b cells, which were independently associated with the presence of T2D by logistic regression models. In summary, this study shows an unbalanced proinflammatory phenotype of B-cell subsets correlated with glycemia and lipidemia in patients with T2D. Our data provide new insight into chronic activation of the immune system and subclinical inflammation in T2D. Further prospective studies are warranted to confirm our observations.

\section{Introduction}

The natural history of diabetes is characterized by a progressive deterioration of glucose metabolism status from euglycemia through prediabetes to type 2 diabetes (T2D) [1]. Obesity, insulin resistance, and insulin secretory dysfunction play different roles during each stage of T2D progression $[2,3]$. Nutrition, physical activity, and genetics also influence the progression to type 2 diabetes. Chronic systemic inflammation is an important link between obesity, insulin resistance, and T2D [4]. Elevated proinflammatory cytokines are linked with decreased insulin sensitivity, while antiinflammatory cytokine expressions are associated with better glucose status [4-7]. Components of the immune system including macrophages, T cells, neutrophils, and eosinophils have been implicated in adipose tissue inflammation and insulin resistance [8-11]. Recent findings showed that altered immune reactivity accompanies and proceeds in $\mathrm{T} 2 \mathrm{D}[12,13]$. Accumulating evidence demonstrated that $\mathrm{B}$ lymphocytes are recruited to adipose tissue in diet-induced obese (DIO) mice and facilitate insulin resistance through proinflammatory $\mathrm{T}$ cells and production of pathogenic IgG antibodies [14]. The activation of $B$ cells is also enhanced in patients with T2D [15]. Furthermore, B cells support T cell-mediated inflammation in subjects with obesity and T2D [16].

$\mathrm{B}$ cells can be divided into two subsets, B-1 and B-2 cells; B-1 cells can be further classified as B-1a and B-1b cells $[17,18]$. It remains controversial about the role of 
TABLe 1: Anthropometric and metabolic data of subjects according to glucose tolerance.

\begin{tabular}{lccc}
\hline & NGT $(n=169)$ & IGR $(n=73)$ & T2D $(n=60)$ \\
\hline Male $(\%)$ & $40.2(68 / 169)$ & $38.4(28 / 73)$ & $46.7(28 / 60)$ \\
Age $(\mathrm{y})$ & $46.38 \pm 11.07$ & $49.86 \pm 11.07$ & $47.75 \pm 13.52$ \\
BMI $\left(\mathrm{kg} / \mathrm{m}^{2}\right)$ & $23.79 \pm 3.31$ & $24.26 \pm 2.92$ & $24.33 \pm 3.36$ \\
WC $(\mathrm{cm})$ & $82.23 \pm 10.52$ & $84.93 \pm 9.01$ & $85.48 \pm 8.18^{*}$ \\
WHR & $0.85 \pm 0.06$ & $0.87 \pm 0.06^{*}$ & $0.88 \pm 0.05^{* *}$ \\
SBP $(\mathrm{mmHg})$ & $116.09 \pm 15.07$ & $122.59 \pm 16.90^{*}$ & $128.50 \pm 16.30^{* *}$ \\
DBP $(\mathrm{mmHg})$ & $75.68 \pm 11.12$ & $78.80 \pm 11.93$ & $84.15 \pm 9.37^{* *}$ \\
TG $(\mathrm{mmol} / \mathrm{L})^{\S}$ & $1.16(0.78-1.78)$ & $1.49(1.08-2.06)^{*}$ & $2.03(0.89-2.97)^{* * *}$ \\
TC $(\mathrm{mmol} / \mathrm{L})$ & $5.09 \pm 0.96$ & $5.03 \pm 0.95$ & $5.12 \pm 1.16$ \\
LDL-C $(\mathrm{mmol} / \mathrm{L})$ & $2.85 \pm 0.86$ & $3.12 \pm 0.80$ & $3.06 \pm 0.82$ \\
HDL-C $(\mathrm{mmol} / \mathrm{L})$ & $1.36(1.11-1.59)$ & $1.26(1.09-1.46)^{*}$ & $1.09(0.80-1.31)^{* * * \dagger}$ \\
HbAlc $(\%)$ & NA & $\mathrm{NA}$ & $6.70(5.60-8.50)$ \\
HbAlc $(\mathrm{mmol} / \mathrm{L})$ & $\mathrm{NA}$ & $\mathrm{NA}$ & $50(38-69)$ \\
FPG $(\mathrm{mmol} / \mathrm{L})^{g}$ & $5.20(4.80-5.50)$ & $5.70(5.20-6.20)^{* * *}$ & $7.19(5.71-8.79)^{* * * \dagger \dagger \dagger}$ \\
FCP $(\mathrm{mmol} / \mathrm{L})^{g}$ & $0.45(0.34-0.63)$ & $0.55(0.45-0.73)^{* *}$ & $0.64(0.37-0.79)^{*}$ \\
\hline
\end{tabular}

Data are $\%(n)$, mean $\pm \mathrm{SD}$, and median (25th-75th percentile). ${ }^{9}$ Compared after Log transformation. ${ }^{\circ}$ Compared by the nonparametric Kruskal-Wallis test; IGR: impaired glucose regulation subjects; N/A: not appropriate; NGT: normal glucose tolerance subjects; T2D: type 2 diabetic subjects; BMI: body mass index; DBP: diastolic blood pressure; FCP: fasting C-peptide; FPG: fasting plasma glucose; HbAlc: glycated hemoglobin; HDL-C: high-density lipoprotein cholesterol; LDL-C: low-density lipoprotein cholesterol; TC: total cholesterol; TG: triglycerides; SBP: systolic blood pressure; WC: waist circumference; WHR: waist-hip ratio; ${ }^{*} P<0.05$ compared with that in the NGT, ${ }^{* *} P<0.01$ compared with that in the NGT, ${ }^{* * *} P<0.001$ compared with that in the NGT, ${ }^{\dagger} P<0.05$ compared with that in the IGR, and ${ }^{\dagger \dagger \dagger} P<0.001$ compared with that in the IGR.

B-cell subsets in obesity and T2D. A recent study demonstrated that B-1a cells attenuate insulin resistance and glucose metabolism through anti-inflammatory cytokine interleukin- (IL-) 10 and polyclonal IgM-dependent mechanisms, while B-2 cells exacerbate metabolic disease [19]. Harmon et al. reported that B-1b cells protect against obesity-associated inflammation and glucose intolerance through IgM mechanism in DIO mice [20]. IL-10producing B (B10) cells, namely, regulatory B cells, control T-cell responses and suppress inflammation via IL-10 [21, 22]. Depletion of B-cell-specific IL-10 deteriorated adipose tissue inflammation and insulin resistance in DIO mice, whereas adoptive transfusion of adipose tissue IL-10-producing regulatory B cells improved those effects [23].

A recent study showed distinct immune cell phenotypes in T2D patients, which were associated with metabolic parameter [24]. Grossmann et al. reported that the immune and inflammatory profiles could be distinguished in each stage of T2D [25]. Of note, high white blood cell count predicts the development of T2D [26]. However, there is no study to investigate the role of B-cell subsets in the development and progression of T2D, especially in the early preclinical stage of disease. In our current study, we compared B-cell compartments in subjects with impaired glucose regulation (IGR) and $\mathrm{T} 2 \mathrm{D}$ with those in normal glucose tolerance (NGT) subjects. We aimed to characterize the phenotype and frequency of B-lymphocyte subsets and their relationship with metabolic factors and disease status.

\section{Materials and Methods}

2.1. Participants. Parts of this study were presented as a poster at the 20th Annual Meeting of the Chinese Diabetes
Society, Xiamen, China, 16-19 Nov 2016 [27]. Subjects with recently diagnosed T2D $(n=60)$ and IGR $(n=73)$ and age-, gender-, and BMI-matched controls with NGT $(n=169)$ (Table 1) were enrolled in the present study from the Second Xiangya Hospital of Central South University. The study was designed in accordance with the principle of the revised Helsinki protocol and approved by the Ethics Committees of the Second Xiangya Hospital of Central South University. All study participants gave written informed consent. Anthropometric and metabolic data were collected by experienced physicians.

Subjects underwent a standardized $75 \mathrm{~g}$ oral glucose tolerance test to investigate glucose status. Study participants were categorized according to WHO criteria, that is, fasting plasma glucose $(\mathrm{FPG})<6.1 \mathrm{mmol} / \mathrm{L}$ and 2 hour plasma glucose $<7.8 \mathrm{mmol} / \mathrm{L}$ as $\mathrm{NGT}$ and $\mathrm{FPG} \geq 6.1 \mathrm{mmol} / \mathrm{L}$ or 2 hour plasma glucose $\geq 7.8 \mathrm{mmol} / \mathrm{L}$ as IGR or prediabetes. Inclusive criteria of T2D were as follows: (1) with a typical history of hyperglycemia; (2) negative for any islet autoantibodies (glutamic acid decarboxylase antibody (GADA), insulinoma-associated protein-2 antibody (IA-2A), and zinc transporter 8 antibody (ZnT8A)); and (3) disease duration $<1$ year, and not on insulin treatment.

Exclusion criteria were (1) type 1 diabetes or secondary diabetes mellitus; (2) any malignant disease, autoimmune disease, inflammation, and infectious disease; (3) history of immunosuppressive medication or steroids for more than 7 days; and (4) pregnant women.

2.2. Laboratory Measurements. A fresh fasting venous blood sample was obtained for determination of glycemia, fasting C-peptide (FCP), total cholesterol (TC), triglycerides (TG), low-density lipoprotein cholesterol (LDL-C), and 
high-density lipoprotein cholesterol (HDL-C). FCP was detected by a chemiluminescence assay (Adiva Centaur System). HbAlc was assessed by high-performance liquid chromatography (Bio-Rad Laboratories). The islet autoantibodies were evaluated by radioligand assays as previously described [28].

2.3. Flow Cytometry. The peripheral blood mononuclear cells were isolated by standard Ficoll-Paque Plus density-gradient centrifugation, and the following monoclonal antibodies were used for human fluorescence-activated cell staining according to the manufacturer's instruction: APC-Cy7-conjugated antihuman CD19 (HIB19), PE-Cy7-conjugated anti-human CD5 (LT21), APC-conjugated anti-human CD1d (51.1), and PerCP-Cy5.5-conjugated anti-human CD23 (EBVCS-5), all from Biolegend. Cell acquisition was performed by using a BD Canto II system and analyzed with FlowJo software as described previously [28]. In brief, total B cells were gated in a SSC/CD19 dot plot. B-cell subsets were defined according to the surface marker expression as $\mathrm{CD} 19^{+}$ $\mathrm{CD} 23^{+}$(B-2), CD19 ${ }^{+} \mathrm{CD} 23^{-}$(B-1), $\mathrm{CD} 19^{+} \mathrm{CD} 23^{-} \mathrm{CD}^{+}$(B1a), $\mathrm{CD} 19^{+} \mathrm{CD} 23^{-} \mathrm{CD}^{-}$(B-1b), and $\mathrm{CD} 19^{+} \mathrm{CD}^{+} \mathrm{CD} 1 \mathrm{~d}^{\text {hi }}$ (B10) cells.

2.4. Statistical Analysis. Data are presented as means \pm standard deviation or as median values (25th-75th percentiles). Statistical analyses were performed using ANOVA or the nonparametric Kruskal-Wallis test for multiple group comparisons. The categorical variables were evaluated by $\chi^{2}$ tests. Spearman's correlations or Pearson's correlations were employed to evaluate associations of the percentages of B-cell subsets with clinical parameters. To assess the effects of B-cell subsets on the presence of IGR and T2D, multinomial logistic regression models were applied to adjust for confounding variables. Adjusted odds ratios (ORs) were provided with 95\% CI and $P$ value. Receiver operating characteristic (ROC) curves and the area under the curve (AUC) were calculated to evaluate performance of B-cell subsets in distinguishing T2D. SPSS 19.0 (IBM Corporation, Chicago, IL) and GraphPad Prism 5 (GraphPad Software, San Diego, CA) were used for data analysis. Two-tailed $P$ value $<0.05$ was considered statistically significant.

\section{Results}

3.1. Anthropometric and Metabolic Characteristics. The baseline characteristics of NGT, IGR, and T2D subjects in groups are presented in Table 1. There were no significant differences in the age, gender, and BMI among the three groups. Patients with T2D had higher waist circumference (WC) and diastolic blood pressure than those of the NGT controls. The levels of waist-hip ratio (WHR), systolic blood pressure (SBP), and TG in subjects with T2D and IGR were elevated than those in NGT controls. There was a gradual increase in the FPG but a gradual decrease in the HDL-C level from NGT controls via individuals with IGR to T2D. The FCP level increased with IGR and remained stable despite progression to $\mathrm{T} 2 \mathrm{D}$.
3.2. Altered Phenotype of B-Cell Subsets. Comparison of frequencies of B-cell subsets among NGT controls, subjects with IGR and T2D, are showed in Figures 1 and 2. There was no significant difference in the frequency of $\mathrm{CD} 19^{+}$and B-1a cells across different glucose status ( $P>0.05$ for both). Patients with T2D had higher B-2 cells (T2D versus NGT, $P<0.01$; T2D versus IGR, $P<0.001$ ) (Figure 2(a)), but T2D patients had lower B-1 cells compared with those of NGT and IGR subjects $(P<0.001$ for both, Figure $2(b))$. The proportion of $\mathrm{B}-1 \mathrm{~b}$ cells tended to be increased in individuals with IGR $(P=0.084)$ and was decreased in patients with T2D $(P<0.001$, Figure $2(\mathrm{c}))$ compared with those in the NGT group. B10 cells were comparable among individuals with NGT and IGR or T2D group and reduced from IGR to T2D (T2D versus NGT, IGR versus NGT, $P>0.05$ for both; T2D versus IGR, $P<0.05$ ) (Figure 2(d)). The frequencies of B-cell subtypes were comparable between IGR and NGT subjects and differed from IGR to T2D (Figure 2).

We also presented the percentages of B-cell subtypes in total PBMCs (see Supplementary Figure 1 available online at https://doi.org/10.1155/2017/5052812). Our results presented above remained the same for the percentages of CD19+, B-2, B-1, B-1a, and B-1b cells among different glucose tolerance groups. However, the percentage of B10 cells did not differ between groups.

3.3. B-Cell Subsets Associated with Metabolic Parameter and Disease Status. The correlations between B-cell subsets and clinical parameters regardless of glucose status are summarized in Table 2. Total B cells associated positively with LDL-C and negatively with HDL-C and FCP. The percentage of B-2 cells was negatively correlated, and the percentage of B-1 cells was positively correlated with female. Of note, $\mathrm{HbAlc}$ and $\mathrm{TG}$ exhibited a positive correlation with B-2 cells but an inverse correlation with B-1 and B- $1 \mathrm{~b}$ cells.

Given that association between gender with B-1 and B-2 cells, we compared the B-cell subsets between male and female in different glucose tolerance groups (Supplementary Figure 2). We found that the proportion of B-1 cells was significantly higher among females than among males in subjects with the NGT and IGR groups, but not with the T2D group. Other B-cell subsets were comparable between male and female in each group. Besides, we also examined the correlations between B-cell subsets and anthropometric data in different glucose tolerance status. In NGT group, the proportion of $\mathrm{CD} 19^{+} \mathrm{B}$ cells associated negatively with FCP $(r=-0.159, P<0.05)$ and positively with LDL-C $(r=0.172, P<0.05)$. BMI showed a positive correlation with B-2 cells $(r=0.187, P<0.05)$ but a negative correlation with B-1 cells $(r=-0.141, P<0.05)$. The B-1b cells exhibited a negative correlation with $\mathrm{HbAlc}(r=-0.164, P<0.01)$. In the IGR group, B-1b cells positively correlated with FCP $(r=0.253, P<0.05)$ and B-2 cells positively correlated with TG $(r=0.140, P<0.05)$. Lastly, in patients with T2D, the percentage of $\mathrm{B}-2$ cells was positively associated with SBP $(r=0.492, P<0.05)$, TG $(r=0.148, P<0.05)$, and HbAlc (Table 2), whereas B-1 and B-1b cells showed a 

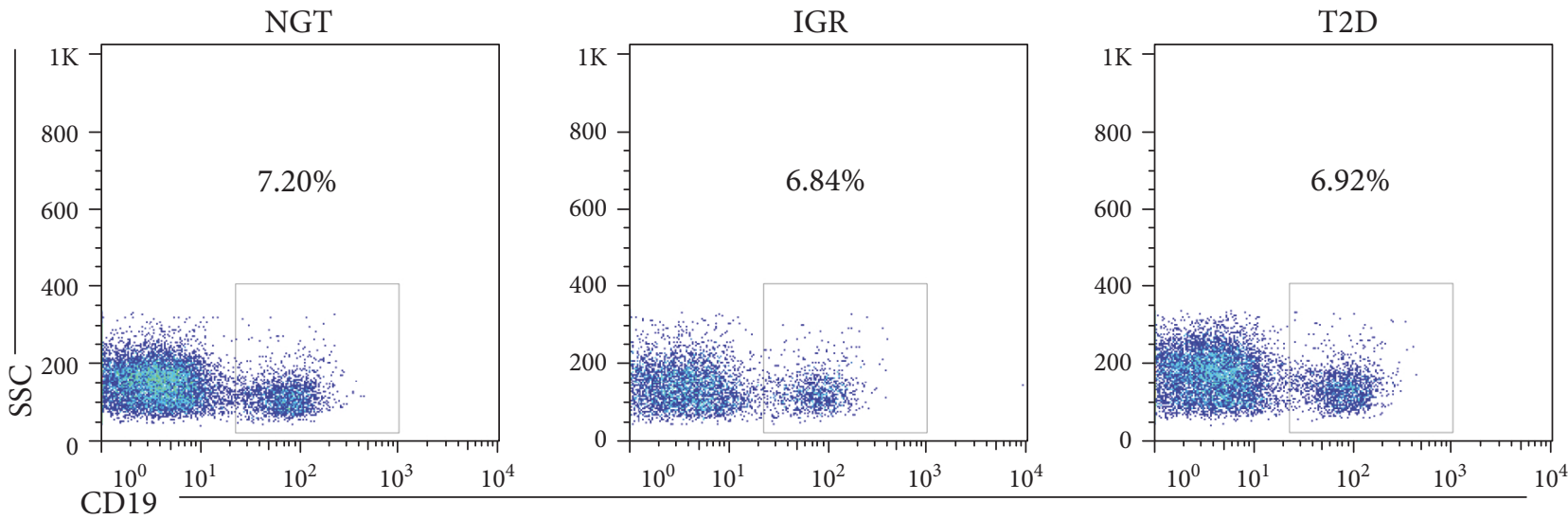

(a)
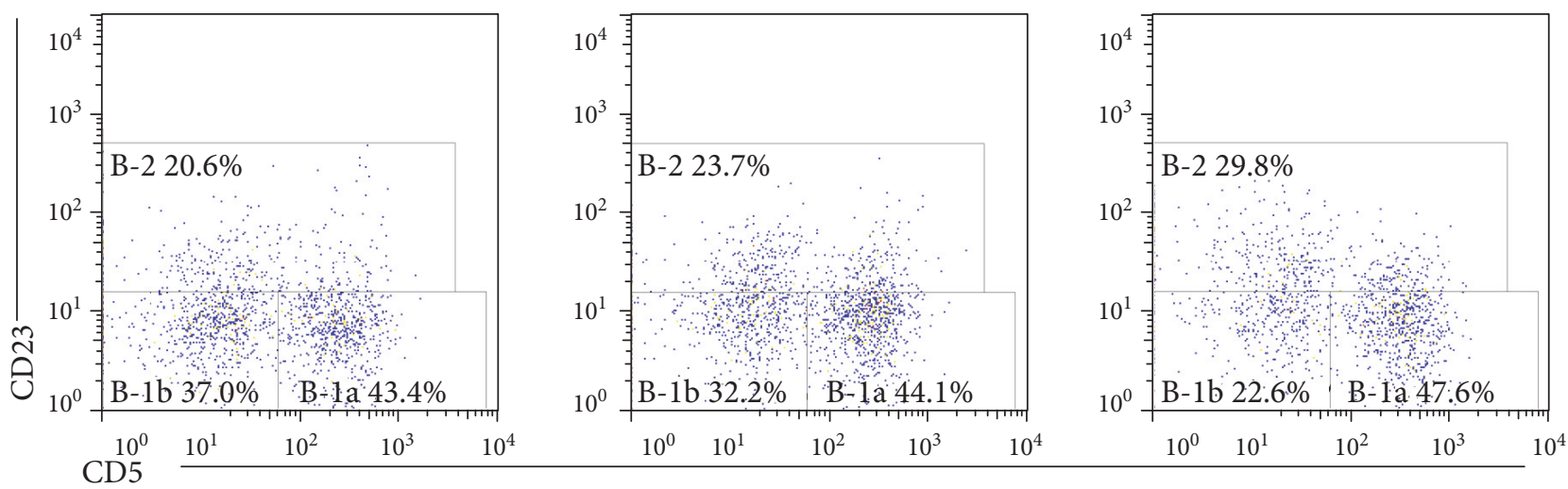

(b)
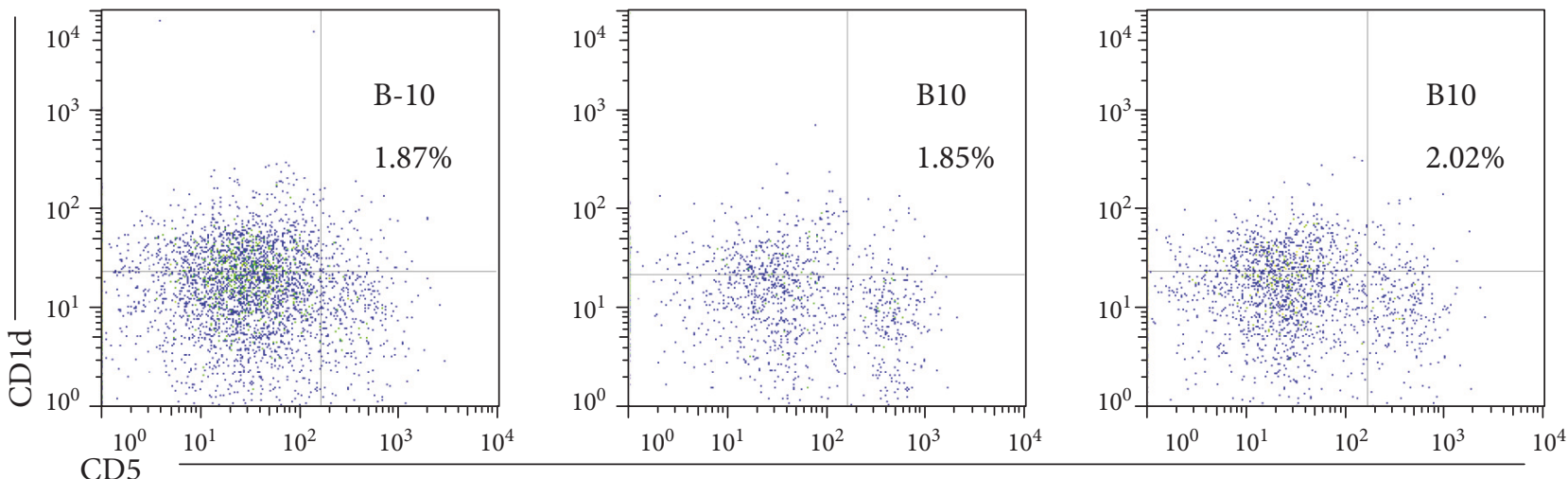

(c)

FIGURE 1: Representative flow cytometry analysis of peripheral B-cell subsets in subjects with NGT, IGR, and T2D. (a) Dots represent CD19+ B-cell frequency in total lymphocytes. Dead cells were excluded from the analysis based on their forward- and side-light scatter properties and propidium iodide staining. Doublets were excluded by FSC-A/FSC-H. (b) Representative dot plot showed the gating strategy for B-2 and $\mathrm{B}-1(\mathrm{~B}-1 \mathrm{a}, \mathrm{B}-1 \mathrm{~b})$ cells in the $\mathrm{CD} 19^{+}$gate. (c) Representative dot plot showed the gating strategy for B10 cells gated on $\mathrm{CD} 19^{+} \mathrm{B}$ cells. IGR, impaired glucose regulation subjects; NGT, normal glucose tolerance subjects; T2D, type 2 diabetic subjects.

negative association with TG $(r=-0.106, \quad P<0.05$; $r=-0.183, P<0.05)$ and HbA1c (Table 2). We tried to evaluate the association between $\mathrm{B}-2$ cells and insulin resistance by using the C-peptide HOMA model, which uses plasma C-peptide concentrations to reflect endogenous insulin secretion [29]. We found a positive correlation between B-2 cells and insulin resistance in subjects with T2D $(r=0.397$,
$P<0.01$ ), whereas there was no association between B-2 cells and insulin resistance in the IGR group.

Multinomial logistic regression analysis of correlations between B-cell subsets and glycemia status is shown in Table 3. The percentages of B-1 and B-1b cells were negatively correlated with $\mathrm{T} 2 \mathrm{D}$ independent from WC, SBP, and TG, although there were no differences for CD $19^{+}$ 


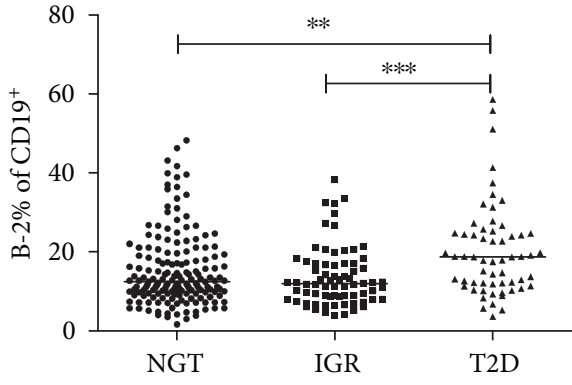

(a)

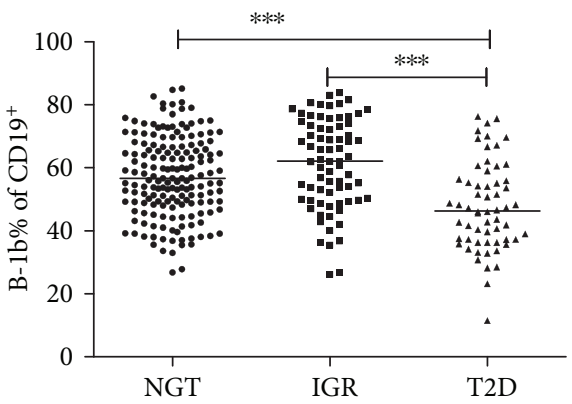

(c)

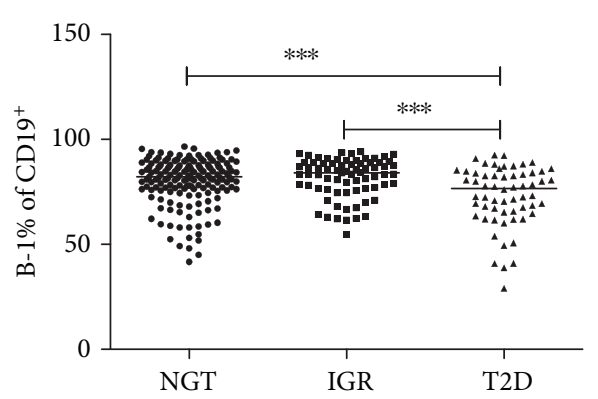

(b)

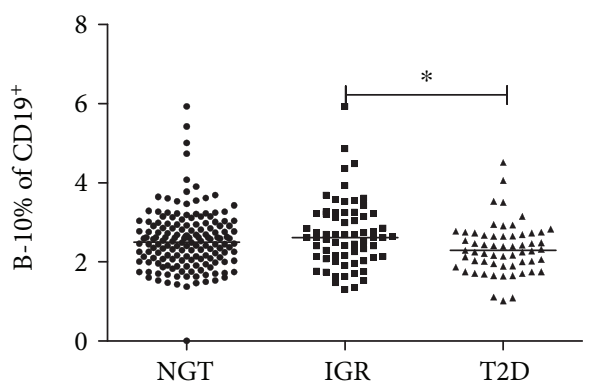

(d)

Figure 2: The frequency of B-cell subsets in subjects of different glucose metabolism status. The frequency of B-2 (a), B-1 (b), B-1b (c), and $\mathrm{B} 10$ (d) cells gated on $\mathrm{CD} 19^{+} \mathrm{B}$ cells. Each point represents the proportion of B-cell subsets of an individual. Horizontal lines show medians. ${ }^{*} P<0.05,{ }^{* *} P<0.01$, and ${ }^{* * *} P<0.001$.

TABLE 2: Association between peripheral B-cell subset percentages and anthropometric and metabolic parameter.

\begin{tabular}{|c|c|c|c|c|c|c|}
\hline & CD19 & B-2 & B-1 & B-1a & B-1b & B10 \\
\hline Sex ${ }^{9}$ & 0.036 & $-0.185^{* *}$ & $0.156^{* *}$ & 0.185 & -0.009 & -0.045 \\
\hline Age & 0.052 & 0.012 & 0.002 & -0.032 & 0.072 & -0.029 \\
\hline BMI & -0.016 & 0.041 & -0.043 & -0.118 & 0.094 & 0.031 \\
\hline WC & 0.039 & 0.033 & -0.057 & -0.069 & 0.052 & 0.048 \\
\hline WHR & 0.056 & 0.045 & -0.051 & -0.051 & 0.034 & 0.032 \\
\hline SBP & 0.114 & 0.006 & -0.046 & 0.013 & -0.066 & -0.121 \\
\hline DBP & 0.060 & 0.114 & -0.111 & -0.051 & -0.023 & -0.063 \\
\hline TC & 0.117 & 0.064 & -0.028 & -0.049 & 0.025 & 0.022 \\
\hline $\mathrm{TG}^{\boldsymbol{s}}$ & 0.054 & $0.198^{*}$ & $-0.181^{*}$ & -0.006 & $-0.183^{* *}$ & 0.007 \\
\hline LDL-C & $0.151^{*}$ & 0.027 & -0.023 & -0.108 & 0.082 & 0.048 \\
\hline HDL-C & $-0.126^{*}$ & -0.071 & 0.088 & 0.076 & 0.046 & -0.055 \\
\hline $\mathrm{FPG}^{9}$ & 0.049 & 0.096 & -0.107 & 0.038 & -0.093 & -0.061 \\
\hline $\mathrm{HbAlc} \mathrm{c}^{\mathbf{s}}$ & 0.090 & $0.185^{* *}$ & $-0.204^{* *}$ & 0.093 & $-0.327^{* * *}$ & -0.088 \\
\hline FCP & $-0.127^{*}$ & 0.068 & -0.035 & -0.144 & 0.062 & -0.047 \\
\hline
\end{tabular}

Association analyses between peripheral B-cell subset percentages (after Log transformation) and anthropometric and metabolic parameter were performed using Pearson's test. 'Spearman's correlations. ${ }^{\S}$ This correlation was only present in T2D individuals. ${ }^{*} P<0.05,{ }^{* *} P<0.01$, and ${ }^{* * *} P<0.001$.

B cells and B-1a cells. Of note, B-2 cells showed a positive association with T2D but a negative correlation with IGR. Other B-cell subsets were not associated with IGR after adjustment for WC, SBP, and TG $(P>0.05)$. A further comparison between subjects with T2D and IGR was showed in Table 3. In addition, we plotted ROC curve analysis for discriminating T2D from the total study population (Figure 3). The AUC for B-2 and reciprocal B-1b cells was 0.667 (95\% CI: $0.592-0.741)$ and 0.710 (95\% CI: $0.635-$ $0.785)$, respectively. Taking the imbalance of $\mathrm{B}-2$ and $\mathrm{B}-1 \mathrm{~b}$ cells into account, we further analyzed the AUC for B-2/B$1 \mathrm{~b}$ and the result was 0.705 (95\% CI: 0.633-0.776). 
TABLE 3: Adjusted logistic regression analysis for the presence of T2D and IGR.

\begin{tabular}{lcccccc}
\hline & \multicolumn{2}{c}{ T2D versus NGT } & \multicolumn{2}{c}{ IGR versus NGT } & \multicolumn{2}{c}{ T2D versus IGR } \\
& OR $(95 \%$ CI $)$ & $P$ value & OR $(95 \%$ CI $)$ & $P$ value & OR (95\% CI) & $P$ value \\
\hline CD19 & $1.677(0.892-3.154)$ & 0.109 & $1.305(0.772-2.205)$ & 0.320 & $1.132(0.539-2.380)$ & 0.743 \\
B-2 & $1.703(1.189-3.261)$ & 0.018 & $0.463(0.306-0.701)$ & $2.730 \times 10^{-4}$ & $2.800(1.398-5.607)$ & 0.004 \\
B-1 & $0.119(0.017-0.844)$ & 0.033 & $3.443(0.965-13.756)$ & 0.054 & $0.015(0.001-0.239)$ & 0.003 \\
B-1b & $0.075(0.017-0.342)$ & 0.001 & $3.222(0.931-11.146)$ & 0.065 & $0.052(0.010-0.273)$ & $4.794 \times 10^{-4}$ \\
B-1a & $1.539(0.826-2.867)$ & 0.174 & $1.018(0.628-1.652)$ & 0.941 & $1.598(0.733-3.486)$ & 0.239 \\
B10 & $0.421(0.137-1.291)$ & 0.130 & $1.436(0.856-2.410)$ & 0.170 & $0.357(0.122-1.049)$ & 0.061 \\
\hline
\end{tabular}

The frequency of B-cell subsets has been Log transferred. Adjusted ORs are given with 95\% CI and $P$ value.

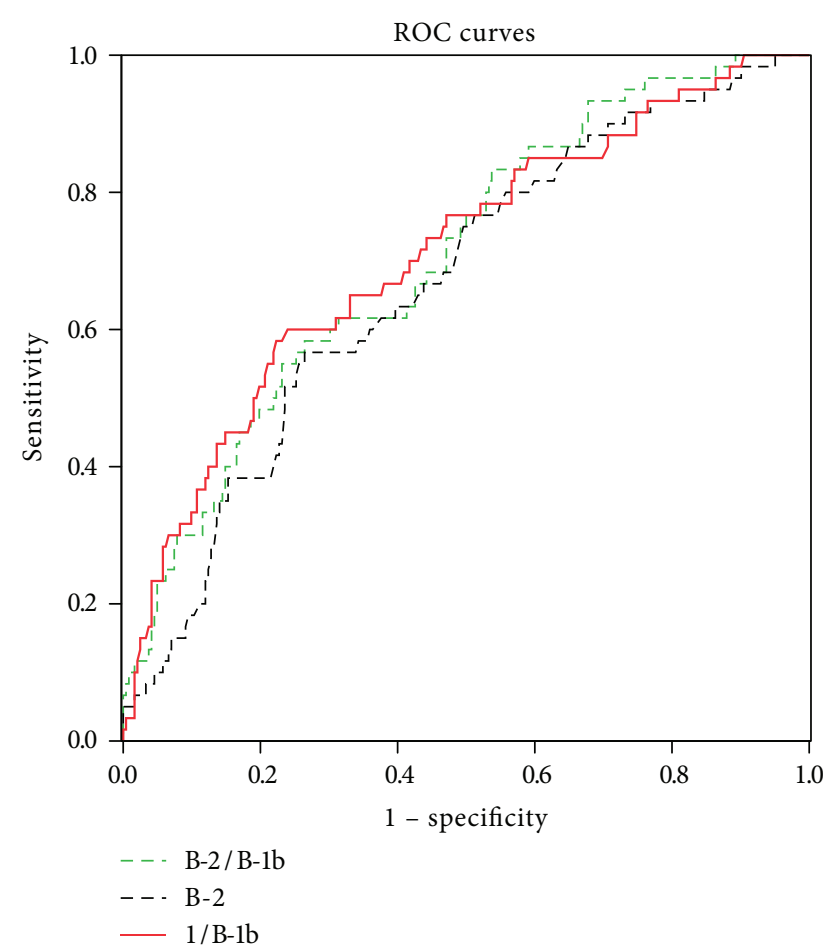

Figure 3: Receiver operating characteristic (ROC) curve analysis of performance of B-cell subsets in distinguishing type 2 diabetes.

\section{Discussion}

A number of studies suggest that B cells are involved in the inflammatory reaction and correlated with insulin resistance in obesity and T2D. To our best knowledge, we for the first time characterize circulating B-cell compartments in subjects with different glucose tolerance status. Our data showed an unbalanced proinflammatory phenotype of B-cell subsets in patients with T2D and their correlation with metabolic characteristics and disease status.

$\mathrm{B}$ cells promote systemic inflammation in DIO mice or/and T2D patients by supporting proinflammatory T-cell function and secretion of IgG antibodies $[14,16]$. Our study found that individuals with T2D had higher B-2 cells compared with those of the NGT and IGR groups (Figure 2(a)) and that B-2 cells were positively correlated with $\mathrm{HbA1c}$ and TG (Table 2). B-2 cells from obese mice produce more proinflammatory cytokine and less IL-10 compared to lean controls [16]. Shen et al. [19] found that high-fat diet (HFD) induced a significant increase in the relative percentage of B-2 cells. Adoptive transfer of B-2 cells exacerbates insulin resistance, whereas depletion of B- 2 cells ameliorates insulin sensitivity in obese mice. IgG antibodies are shown to initiate adipose tissue inflammation and enhance insulin resistance [14]. These IgG antibodies are resident in visceral adipose tissue and secreted by adoptive B-2 cells [30].

Conversely, B cells have also been reported to play important roles in inhibiting inflammation and insulin resistance by IL-10 or/and polyclonal IgM mechanism [19, 20, 23]. Patients with T2D displayed a reduction in B-1 cells compared with those of the NGT and IGR groups, attributable to a reduction in B-1b cells (Figures 2(b) and 2(c)), which both expressed a negative association with HbA1c and TG (Table 2). B-1-derived IgM antibodies can alleviate inflammation and promote apoptotic cell clearance [31], which is impaired during the progress to obesity [32]. B-1a and B-1b cells reveal a unique function under different immunostimulation to $S$. pneumoniae [18]. The protective role of B-1 subsets against the inflammatory and metabolic abnormity of DIO mice remains controversial [19, 20]. HFD induces a significant decrease in the proportion of B-1a cells in the visceral adipose tissue. Remarkably, B-1a-cell transfusion protects against insulin resistance through IL-10 and polyclonal IgM mechanism [19]. Nevertheless, B-1b cells instead of B-1a cells are more frequent in $\mathrm{Id} 3^{\text {Bcell KO }}$ mice and improve adipose tissue inflammation and glucose intolerance in DIO mice by IgM antibodies [20]. In addition, B-1-like cells are identified and accumulated in human omental adipose tissue, and IgM antibodies are negatively associated with inflammation and insulin resistance [20]. Taken together, similar to Th1/Th2 imbalance, the imbalance of B-2 and B-1b cells in total CD19 ${ }^{+} \mathrm{B}$ cells might point to an upregulated proinflammatory status with higher glycemia and lipidemia. A prospective and longitudinal study is warranted to confirm our observations.

We reported unique B10 cells in patients with autoimmune diabetes in our previous publication [28]. In our current study, we did not find any differences in B10 cells between the NGT controls and IGR or T2D group (Figure 2(d)). B10 cells have a regulatory function in inflammation, infection, and autoimmunity [21, 22, 33]. Loss- and gain-function study of B10 cells showed the protective role in adipose tissue inflammation [23]. 
Several phenotypic B-cell subsets are capable of producing IL-10 upon stimulation and of suppressing inflammatory processes in human $[34,35]$ but lack the suppressive capacity in systemic lupus erythematosus patients [34, 35]. Whether the regulatory function of IL-10-producing B cells in T2D is impaired requires further confirmatory experiments in the future study.

We were unable to determine any difference in B-cell subsets between NGT and IGR subjects (Figure 2), which suggested that obesity-related inflammation and insulin resistance might play a more important role in the change of B-cell subsets compared with hyperglycemia, given that the IGR and T2D subjects enrolled in the study were overweight, but not obese. This is in line with previous results that obesity has a substantial effect on the lifetime risk of prediabetes and diabetes [1] and induces a phenotypic switch in adipose tissue macrophage polarization [9]. The percentage of visceral adiposity and the risk factors for cardiovascular diseases are generally higher among Asian individuals compared with white populations with the same BMI or waist circumference. The metabolically obese phenotype of patients who have normal BMI level but increased visceral adiposity has been correlated with high risk of developing T2D [36]. Asians are more genetically susceptible to insulin resistance and diabetes than Western counterparts [36]. Besides, among Asian young patients with early-onset diabetes (age at diagnosis $\leq 40$ years), nearly $40 \%$ had a lean, nonautoimmune phenotype with low levels of $\beta$-cell function [37]. In addition, epigenetic factors including gestational or overt diabetes in pregnancy, low birth weight, exposure to undernutrition in utero, micronutrient imbalances, and gut microbiota might contribute to increased risk for T2D [38, 39].

In the multinomial logistic regression model, B-2 cell was negatively associated with IGR but positively correlated with T2D. In contrast to B-2 cells, B-1b cells decreased in T2D patients and associated negatively with the presence of T2D independent of SBP, WC, and TG. These might suggest vital roles of $\mathrm{B}$-cell subset in the pathogenesis of $\mathrm{T} 2 \mathrm{D}$ and support the concept that metabolic syndrome components, SBP, TG, WC, and hyperglycemia, together affect the immune system in T2D and that the central mechanism of metabolic syndrome is insulin resistance and inflammation. The potential value of $\mathrm{B}$-cell subsets in the determination of T2D was evaluated by ROC analysis. However, the AUC assays did not yield good model performance $(\mathrm{AUC}=0.6670 .710$ ), which was likely due to comparatively large individual variation (Figure 2). Improved methodological aspects and integration with other parameter may optimize the diagnostic value.

Our study shows for the first time distinct frequencies of $\mathrm{B}$-cell subsets in T2D. This provides new insight into the spectrum of immune and inflammatory profiles associated with human T2D $[24,25,40]$. However, this study has also some limitations as follows. This cross-sectional study does not allow us to draw a conclusion regarding a causal interrelation between diabetes, inflammation, and immunity. Besides, we do not address infiltrating immune cells from adipose tissue, serum cytokines, or immunoglobulin levels.

\section{Conclusions}

This study shows the unbalance of B-cell subsets toward proinflammatory phenotype in T2D patients, and the B-cell subsets are correlated with glycemia and lipidemia. Our data provide new insight into chronic activation of immune system and subclinical inflammation in T2D. Further prospective studies as well as in vitro function experiments are warranted to confirm our observations.

\section{Disclosure}

Parts of this study were presented as a poster at the 20th Annual Meeting of the Chinese Diabetes Society, Xiamen, China, 16-19 Nov 2016.

\section{Conflicts of Interest}

No other potential conflicts of interest relevant to this article were reported.

\section{Authors' Contributions}

Chao Deng performed the experiments, analyzed the data, wrote the manuscript, and contributed to the discussion. Yufei Xiang designed the study, researched the data, edited the manuscript, contributed to the discussion, and provided the critical review. Tingting Tan, Zhihui Ren, Chuqing Cao, and Bingwen Liu performed the experiments. Gan Huang contributed to the islet autoantibody assay and discussion. Xiangbing Wang contributed to the discussion and edited the manuscript. Zhiguang Zhou designed the study, contributed to the discussion, and edited the manuscript. Zhiguang Zhou is the guarantor of this work and, as such, had full access to all the data in the study and takes responsibility for the integrity of the data and the accuracy of the data analysis. Chao Deng and Yufei Xiang contributed equally to this study.

\section{Acknowledgments}

This study was supported by the National Clinical Research Center for Metabolic Diseases in China (2013BAI09B12), the Key Project of Chinese Ministry of Education (113050A), and the National Natural Science Foundation of China (81461168031; 81400817). Dr. Yufei Xiang was supported by the European Foundation for the Study of Diabetes (EFSD) fellowship and Shenghua Yuying talented program of Central South University.

\section{References}

[1] S. Ligthart, T. T. van Herpt, M. J. Leening et al., "Lifetime risk of developing impaired glucose metabolism and eventual progression from prediabetes to type 2 diabetes: a prospective cohort study," The Lancet Diabetes and Endocrinology, vol. 4, no. 1, pp. 44-51, 2016.

[2] J. H. Ohn, S. H. Kwak, Y. M. Cho et al., "10-year trajectory of beta-cell function and insulin sensitivity in the development of type 2 diabetes: a community-based prospective 
cohort study," The Lancet Diabetes and Endocrinology, vol. 4, no. 1, pp. 27-34, 2016.

[3] C. Weyer, P. A. Tataranni, C. Bogardus, and R. E. Pratley, "Insulin resistance and insulin secretory dysfunction are independent predictors of worsening of glucose tolerance during each stage of type 2 diabetes development," Diabetes Care, vol. 24, no. 1, pp. 89-94, 2001.

[4] M. Y. Donath and S. E. Shoelson, "Type 2 diabetes as an inflammatory disease," Nature Reviews. Immunology, vol. 11, no. 2, pp. 98-107, 2011.

[5] M. N. Pham, M. I. Hawa, C. Pfleger et al., "Pro- and antiinflammatory cytokines in latent autoimmune diabetes in adults, type 1 and type 2 diabetes patients: action LADA 4," Diabetologia, vol. 54, no. 7, pp. 1630-1638, 2011.

[6] Y. Xiang, P. Zhou, X. Li et al., "Heterogeneity of altered cytokine levels across the clinical spectrum of diabetes in China," Diabetes Care, vol. 34, no. 7, pp. 1639-1641, 2011.

[7] E. G. Hong, H. J. Ko, Y. R. Cho et al., "Interleukin-10 prevents diet-induced insulin resistance by attenuating macrophage and cytokine response in skeletal muscle," Diabetes, vol. 58, no. 11, pp. 2525-2535, 2009.

[8] S. Nishimura, I. Manabe, M. Nagasaki et al., "CD8+ effector T cells contribute to macrophage recruitment and adipose tissue inflammation in obesity," Nature Medicine, vol. 15, no. 8, pp. 914-920, 2009.

[9] C. N. Lumeng, J. L. Bodzin, and A. R. Saltiel, “Obesity induces a phenotypic switch in adipose tissue macrophage polarization," The Journal of Clinical Investigation, vol. 117, no. 1, pp. 175-184, 2007.

[10] S. Talukdar, D. Y. Oh, G. Bandyopadhyay et al., "Neutrophils mediate insulin resistance in mice fed a high-fat diet through secreted elastase," Nature Medicine, vol. 18, no. 9, pp. 1407-1412, 2012.

[11] D. Wu, A. B. Molofsky, H. E. Liang et al., "Eosinophils sustain adipose alternatively activated macrophages associated with glucose homeostasis," Science, vol. 332, no. 6026, pp. 243-247, 2011.

[12] C. Herder, J. Baumert, B. Thorand et al., "Chemokines as risk factors for type 2 diabetes: results from the MONICA/KORA Augsburg study, 1984-2002," Diabetologia, vol. 49, no. 5, pp. 921-929, 2006.

[13] H. Sell, C. Habich, and J. Eckel, "Adaptive immunity in obesity and insulin resistance," Nature Reviews. Endocrinology, vol. 8, no. 12, pp. 709-716, 2012.

[14] D. A. Winer, S. Winer, L. Shen et al., "B cells promote insulin resistance through modulation of $\mathrm{T}$ cells and production of pathogenic IgG antibodies," Nature Medicine, vol. 17, no. 5, pp. 610-617, 2011.

[15] M. Jagannathan, M. McDonnell, Y. Liang et al., "Toll-like receptors regulate $\mathrm{B}$ cell cytokine production in patients with diabetes," Diabetologia, vol. 53, no. 7, pp. 1461$1471,2010$.

[16] J. DeFuria, A. C. Belkina, M. Jagannathan-Bogdan et al., "B cells promote inflammation in obesity and type 2 diabetes through regulation of T-cell function and an inflammatory cytokine profile," Proceedings of the National Academy of Sciences of the United States of America, vol. 110, no. 13, pp. 5133-5138, 2013.

[17] N. Baumgarth, "The double life of a B-1 cell: self-reactivity selects for protective effector functions," Nature Reviews. Immunology, vol. 11, no. 1, pp. 34-46, 2011.
[18] K. M. Haas, J. C. Poe, D. A. Steeber, and T. F. Tedder, "B-1a and B-1b cells exhibit distinct developmental requirements and have unique functional roles in innate and adaptive immunity to S. Pneumoniae," Immunity, vol. 23, no. 1, pp. 7-18, 2005.

[19] L. Shen, M. H. Chng, M. N. Alonso, R. Yuan, D. A. Winer, and E. G. Engleman, "B-1a lymphocytes attenuate insulin resistance," Diabetes, vol. 64, no. 2, pp. 593-603, 2015.

[20] D. B. Harmon, P. Srikakulapu, J. L. Kaplan et al., "Protective role for B-1b B cells and IgM in obesity-associated inflammation, glucose intolerance, and insulin resistance," Arteriosclerosis, Thrombosis, and Vascular Biology, vol. 36, no. 4, pp. 682-691, 2016.

[21] S. Amu, S. P. Saunders, M. Kronenberg, N. E. Mangan, A. Atzberger, and P. G. Fallon, "Regulatory B cells prevent and reverse allergic airway inflammation via FoxP3positive T regulatory cells in a murine model," The Journal of Allergy and Clinical Immunology, vol. 125, no. 5, pp. 1114$1124,2010$.

[22] K. Yanaba, J. D. Bouaziz, K. M. Haas, J. C. Poe, M. Fujimoto, and T. F. Tedder, "A regulatory $B$ cell subset with a unique CD1dhiCD5+ phenotype controls $\mathrm{T}$ cell-dependent inflammatory responses," Immunity, vol. 28, no. 5, pp. 639650, 2008.

[23] S. Nishimura, I. Manabe, S. Takaki et al., "Adipose natural regulatory B cells negatively control adipose tissue inflammation," Cell Metabolism, vol. 18, no. 5, pp. 759-766, 2013.

[24] B. Menart-Houtermans, R. Rutter, B. Nowotny et al., "Leukocyte profiles differ between type 1 and type 2 diabetes and are associated with metabolic phenotypes: results from the German diabetes study (GDS)," Diabetes Care, vol. 37, no. 8, pp. 2326-2333, 2014.

[25] V. Grossmann, V. H. Schmitt, T. Zeller et al., "Profile of the immune and inflammatory response in individuals with prediabetes and type 2 diabetes," Diabetes Care, vol. 38, no. 7, pp. 1356-1364, 2015.

[26] B. Vozarova, C. Weyer, R. S. Lindsay, R. E. Pratley, C. Bogardus, and P. A. Tataranni, "High white blood cell count is associated with a worsening of insulin sensitivity and predicts the development of type 2 diabetes," Diabetes, vol. 51, no. 2, pp. 455-461, 2002.

[27] Z. Zhou, Y. Xiang, L. Ji et al., "Frequency, immunogenetics, and clinical characteristics of latent autoimmune diabetes in China (LADA China study): a nationwide, multicenter, clinic-based cross-sectional study," Diabetes, vol. 62, no. 2, pp. 543-550, 2013.

[28] C. Deng, Y. Xiang, T. Tan et al., "Altered peripheral Blymphocyte subsets in type 1 diabetes and latent autoimmune diabetes in adults," Diabetes Care, vol. 39, no. 3, pp. 434-440, 2016.

[29] T. M. Wallace, J. C. Levy, and D. R. Matthews, "Use and abuse of HOMA modeling," Diabetes Care, vol. 27, no. 6, pp. 14871495, 2004.

[30] K. Rajewsky, "Clonal selection and learning in the antibody system,” Nature, vol. 381, no. 6585, pp. 751-758, 1996.

[31] M. Y. Chou, L. Fogelstrand, K. Hartvigsen et al., "Oxidationspecific epitopes are dominant targets of innate natural antibodies in mice and humans," The Journal of Clinical Investigation, vol. 119, no. 5, pp. 1335-1349, 2009.

[32] S. Cinti, G. Mitchell, G. Barbatelli et al., "Adipocyte death defines macrophage localization and function in adipose tissue 
of obese mice and humans," Journal of Lipid Research, vol. 46, no. 11, pp. 2347-2355, 2005.

[33] S. Fillatreau, C. H. Sweenie, M. J. McGeachy, D. Gray, and S. M. Anderton, "B cells regulate autoimmunity by provision of IL-10," Nature Immunology, vol. 3, no. 10, pp. 944-950, 2002.

[34] Y. Iwata, T. Matsushita, M. Horikawa et al., "Characterization of a rare IL-10-competent B-cell subset in humans that parallels mouse regulatory B10 cells," Blood, vol. 117, no. 2, pp. 530-541, 2011.

[35] P. A. Blair, L. Y. Noreña, F. Flores-Borja et al., "CD19+CD24hiCD38hi B cells exhibit regulatory capacity in healthy individuals but are functionally impaired in systemic lupus erythematosus patients," Immunity, vol. 32, no. 1, pp. 129-140, 2010.

[36] S. A. Lear, K. H. Humphries, S. Kohli, A. Chockalingam, J. J. Frohlich, and C. L. Birmingham, "Visceral adipose tissue accumulation differs according to ethnic background: results of the multicultural community health assessment trial (M-CHAT)," The American Journal of Clinical Nutrition, vol. 86, no. 2, pp. 353-359, 2007.

[37] M. C. Ng, S. C. Lee, G. T. Ko et al., "Familial early-onset type 2 diabetes in Chinese patients: obesity and genetics have more significant roles than autoimmunity," Diabetes Care, vol. 24, no. 4, pp. 663-671, 2001.

[38] C. J. Nolan, P. Damm, and M. Prentki, “Type 2 diabetes across generations: from pathophysiology to prevention and management," Lancet, vol. 378, no. 9786, pp. 169-181, 2011.

[39] J. C. Chan, V. Malik, W. Jia et al., "Diabetes in Asia: epidemiology, risk factors, and pathophysiology," Jama, vol. 301, no. 20, pp. 2129-2140, 2009.

[40] C. Zeng, X. Shi, B. Zhang et al., “The imbalance of Th17/Th1/ Tregs in patients with type 2 diabetes: relationship with metabolic factors and complications," Journal of Molecular Medicine (Berlin, Germany), vol. 90, no. 2, pp. 175-186, 2012. 


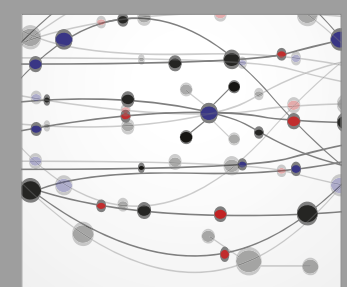

The Scientific World Journal
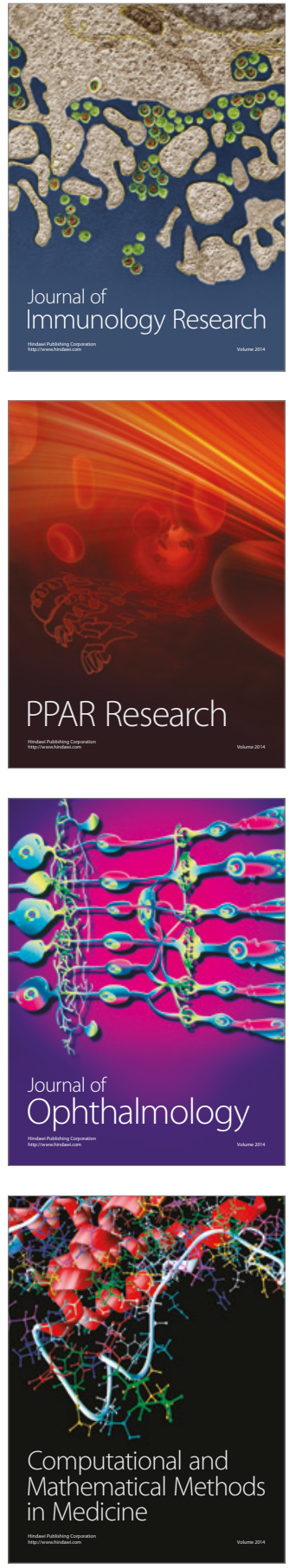

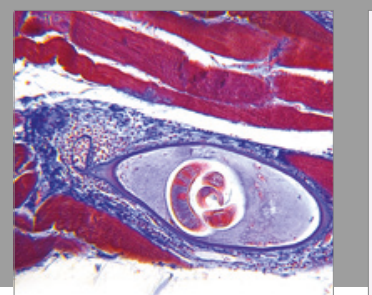

Gastroenterology Research and Practice
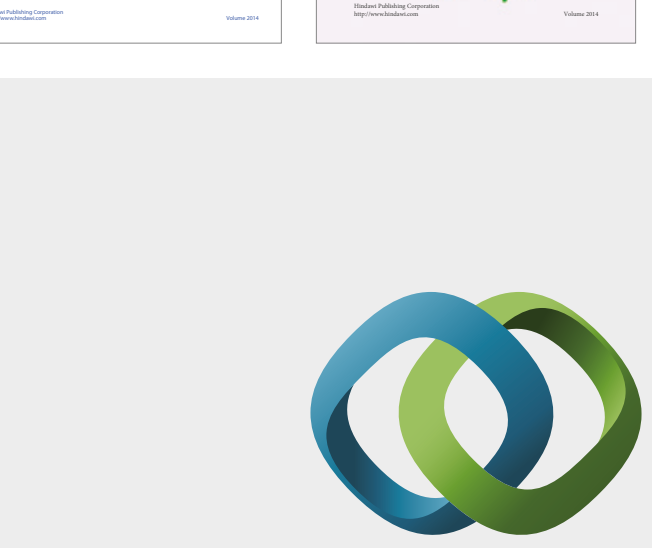

\section{Hindawi}

Submit your manuscripts at

https://www.hindawi.com
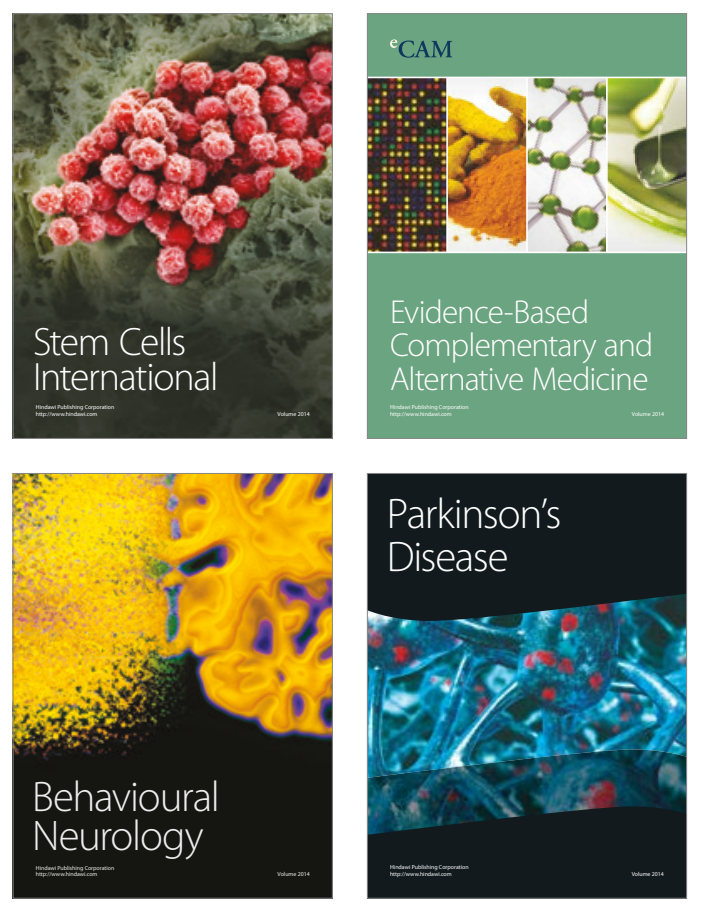
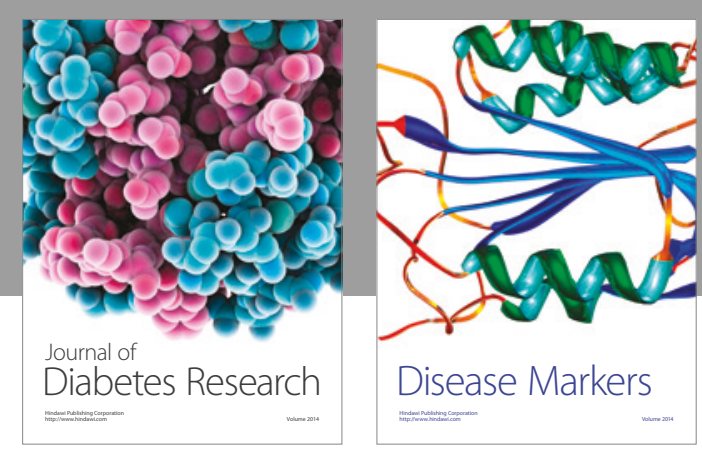

Disease Markers
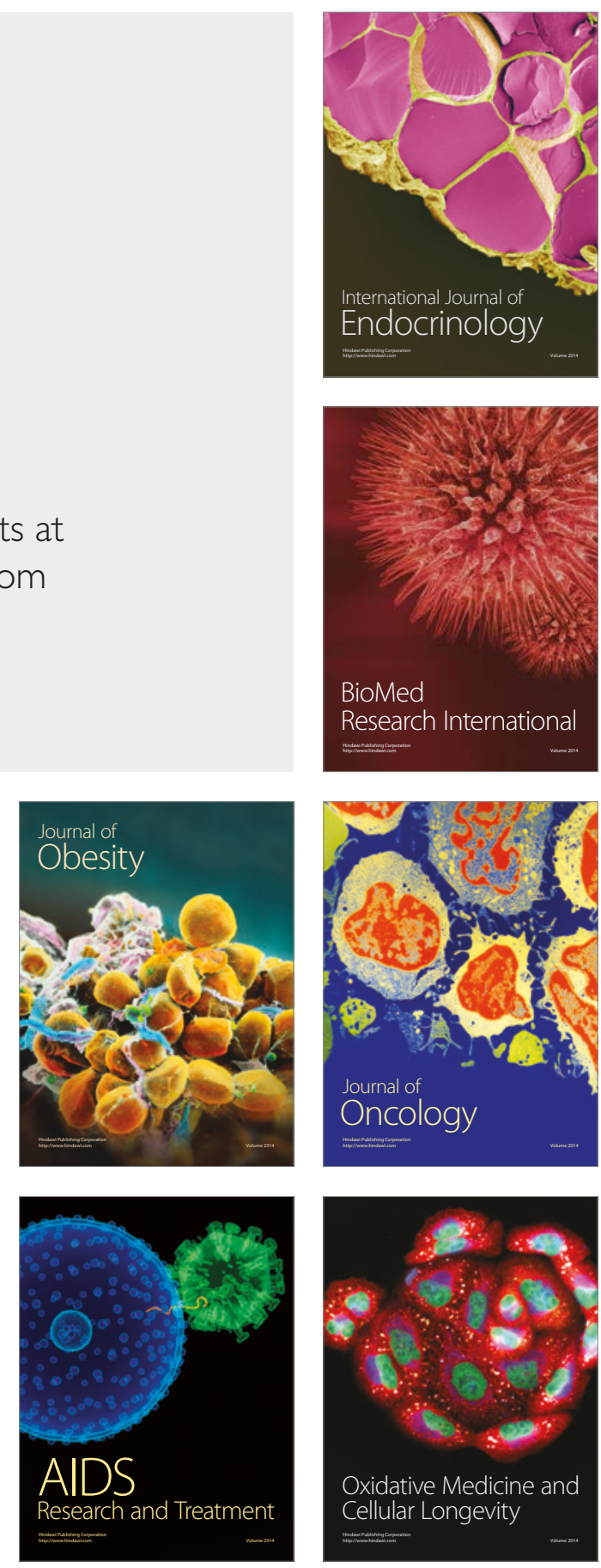